\title{
The Internet of Services: Global Industrialization of Information Intensive Services
}

\author{
Christoph Schroth \\ SAP Research CEC St. Gallen, Switzerland \\ University of St. Gallen, MCM Institute, Switzerland \\ christoph.schroth@sap.com
}

\begin{abstract}
Web Services have experienced great interest during the last years as they were expected to act as enablers of seamless application-to-application integration both within company boundaries and on a global scale. However, a truly global mesh of interoperable services has not yet become reality due to various reasons. Novel technologies and design principles are now about to emerge which shift the Internet towards a major inflection point: It is envisioned to evolve as a global platform for the retrieval, combination and utilization of interoperable resources. In this article, we contrast the Internet of today and the envisioned Internet of Services along three major categories: Stakeholders, Applications and finally Technology. We conclude with an analysis of the important role this emerging Internet of Services will play as technological enabler of information services industrialization: By reducing different kinds of waste, the Internet of Services acts as enabler of Lean Service Management
\end{abstract}

\section{Introduction}

Web Services have experienced great interest during the last years as they were expected to play a key role as technical basis for the realization of ServiceOriented Architectures (SOAs). However, Web Services-based SOAs still mostly exist within company boundaries [1]. The global provision and consumption of services via the Internet is still at an early stage and have not yet taken over a significant role in realizing cross-organizational collaboration. Various reasons such as high technical complexity, implementation and maintenance costs, inflexibility and the lack of widely accepted standards for defining service choreographies as well as message semantics have been repeatedly identified as key factors that have prevented the emergence of a global mesh of interoperable Web Services [2]. Further hurdles on the path to a "Global SOA" include the lack of globalscale service discovery as well as platforms allowing for intuitive human-guided service interaction and composition. The recent emergence of the Web 2.0 [3, 4] phenomenon has been hoped to remove some of these challenges: Its focus on user-empowerment, the promoted relevance of rich user experience and the consideration of the Web as platform for building systems certainly facilitates the establishment of global service-orientation.

In this work, we elaborate on how principles and technologies from both Web 2.0 and SOA can be used to facilitate the emergence of a "Global SOA". We propose the Internet of Services (IoS) reference architecture which considers the Internet as a global platform for the retrieval, combination and utilization of interoperable resources. This next-generation Internet will allow both private users and companies to seamlessly collaborate through the use of interoperable, electronic services and thus drive the industrialization of information intensive services.

The remainder of the paper is structured as follows: In Chapter 2 we first of all revisit the notion of SOA and analyze its major shortcomings with regard to the emergence of a Global SOA. In Chapter 3, we present a novel reference architecture of an IoS which concerns the levels stakeholders, applications as well as technology. Finally, its expected impact with respect to the world-wide industrialization of information intensive services is analyzed in Chapter 4. Chapter 5 concludes this work with a brief summary and outlook.

\section{The Web Services stack and its shortcomings on the path towards a global SOA}

Since the late 1990s, many definitions of SOA have been published [5]. The widely accepted normative OASIS Reference Model for SOA [6] defines SOA as "...a paradigm for organizing and utilizing distributed capabilities that may be under the control of different ownership domains. It provides a uniform means to offer, discover, interact with and use capabilities to produce desired effects consistent with measurable preconditions and expectations". According to this 
model, the major components of a basic SOA and their possible interactions are: a service provider publishes his service interface via a service registry where a service requester can find it and subsequently may bind to the service provider. This enables a loose coupling of services (thereby minimizing mutual dependencies) and complies with some of the probably most-known principles in software-engineering, information-hiding and modularization. The concept of supporting loosely coupled, business-aligned and networked services can be realized with the help of numerous different technologies. As WSDL/- SOAPbased Web Services are the most widely spread application of SOA on the Web, we take these as a foundation of our work [5]: The Web Service Description Language (WSDL) thereby defines service interfaces. The Universal Description, Discovery, and Integration (UDDI) standard is leveraged to define publicly available service registries, while the Business Process Execution Language (BPEL) is widely accepted as a standard for orchestrating different services into one process choreography.

Service-Oriented Architectures (SOAs) on the basis of Web Services have not yet lived up to their promise to enable seamless and automated e-Business infrastructures and cross-organizational Enterprise Application Integration (EAI). In fact, implementations are still limited to a company-internal scope. Different challenges exist that prevent them from "growing" beyond corporate boundaries and thus allowing for a global and highly dynamic mesh of interoperable services. One first challenge of cross-organizational application integration that has not yet been solved by the SOA concept is that of semantic interoperability [7]. Web services usually rely on the XML Schema language to determine the structure of documents that are exchanged between the applications [5]. However, it is not possible to capture semantics of message elements with the help of such schemas. Current SOA implementations also usually lack a semantically rich description of the services involved. Automatic discovery and composition of services cannot be realized unless the naming of service properties is unambiguously defined and thus machine-readable. As a further major hurdle, the heterogeneity of the Web Services stack prevents from seamless interoperability. A plethora of different standards has emerged and prevents from simple and efficient setup of inter-organizational SOAs as different services cannot be loosely coupled (to allow for seamless interoperability, the same WS protocol stack implementation must be used). Also, there is no widely accepted service broker or market place that enables the world-wide search for services that match a user's needs. One further important shortcoming of existing SOAs is related to the gap between human users and machines: Web Services are usually used to automate machine-to-machine collaboration but do not feature a "face" to human users as they reside on a merely technical layer. They cannot be easily found (only few and complex registries exist) or understood (interfaces are defined as XML-files which are only comprehendible for experts).

\section{The Internet of Services Reference Architecture}

To allow for the loose coupling across company boundaries on a global scale and to thus realize a "global SOA", new concepts and technologies are required. Recently, the evolution of the Internet towards a global platform for the retrieval, combination and utilization of rich resources has experienced great interest. In this section, today's Internet is contrasted with the envisioned IoS along three major categories (Figure 1) on the basis of case studies: The Stakeholders component describes the characteristics of resource providers, consumers as well as intermediary players and their exact functions. The Applications component accounts for applications management, the types of available resources and the key paradigms of application development. Finally, the Technology component deals with resource composition, resource interfaces as well as messaging protocols.

\subsection{Stakeholders}

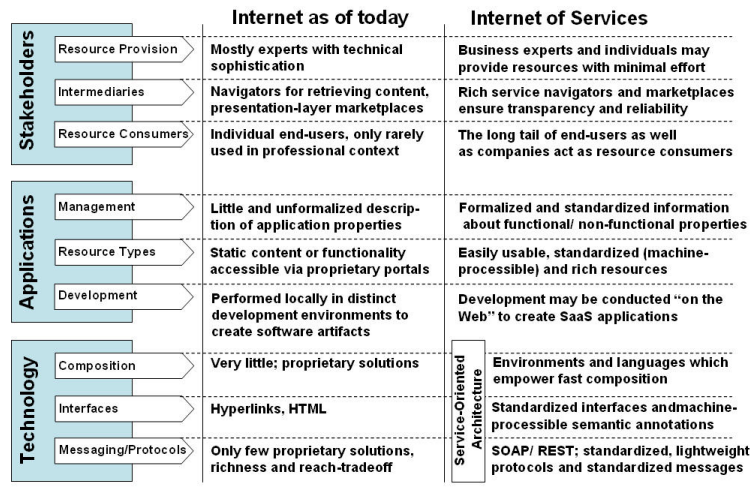

Figure 1: Stakeholders, Applications and Technology in an Internet of Services

Resource Provision. In the Internet we know today, content or functionality is mostly created and provided by experts with technical sophistication. Company portals, Websites managed by newspapers, and many other "island" applications providing services or 
content are usually setup and maintained by people who need specific skills in the field of Web programming. The recent emergence of Web-based platforms, interactive encyclopedias and Web logs, which are often being summarized under the umbrella term Web 2.0, already shows the direction of the future Internet [4]: Web-based platforms are emerging that allow technically unsophisticated end-users to create and subsequently publish, manage and maintain content or even services that can be consumed by arbitrary other stakeholders. In [2], a vision of seamless, cross-organizational collaboration on the basis of a future IoS is drawn: Business experts (not only IT experts) are enabled to publish their company's services on the Web and also consume those of other businesses.

Intermediaries. As described by Evans and Wurster [8], intermediaries represent central enablers of the Internet's effectiveness. So-called navigators act as intermediary "institutions" that provide transparency, guide Internet users and help different stakeholders finding each other (e.g., the search engine Google.com). According to North [9], the reason for the existence of institutions is the uncertainties involved in the interaction of agents; institutions act as constraints and framework to structure that interaction and make it more reliable. The proper establishment and application of informal constraints, formal rules and enforcement mechanisms are adequate to lower transaction costs and boost economic performance. The Google search engine, for example, is recognized as trustworthy central point of access to different kinds of Web-based resources. By ranking search results, the otherwise infinite choice between the different resources available via the Web is limited. New kinds of intermediaries will be crucial for the successful emergence and wide acceptance of an IoS. The readily available Web Services stack [5, 6] foresees publicly available service registries (on the basis of the UDDI standard). However, the UDDI standard aims at technical experts, only specifies very limited functionality and is therefore not adequate to serve the large number of normal Internet users who require intuitive, human-readable interfaces. Novel forms of intermediaries are now about to emerge which offer richer resource registry functionality and extend the role of the traditional UDDI-based imple-mentations:

First, a high degree of transparency is provided for users who desire to find dedicated resources in the Web. Intermediaries do not only contain references to services any more, but also offer statistics about resource performance, availability or information about evaluations conducted by previous users. This significantly reduces uncertainty in a global networked economy and improves predictability of resource provision and consumption. Second, novel intermediaries provide sophisticated navigation functionality. As opposed to existing standards, they allow users to draw on descriptions created by others and to leverage a plethora of functional as well as nonfunctional resource attributes to retrieve content or functionality as efficiently as possible. Governance represents the third service provided by emerging intermediaries in an IoS. In today's Internet, Webbased resources and their mutual relationships can still be considered rather ungoverned. Information as well as functionality can be published and changed according to individual requirements and is only rarely subject to formal agreements between resource providers and consumers. To facilitate the emergence of an IoS, service management concepts such as governance procedures which have successfully been deployed within corporate boundaries [10] must be extended towards the Internet. Independent institutions will have to be established which control and enforce compliance of both resource providers and consumers with respect to their previously established agreements. Only on the basis of a solid institutional groundwork, companies will decide to use this next-generation Internet for offering and consuming mission-critical resources over the Web. Therefore, the intermediaries introduced above will have to, besides of acting as rich service registries, also guarantee for predictability and dependability of electronic services. The support of negotiation, brokering, translation and monitoring of Service-Level Agreements will become crucial tasks for future institutions to increase productivity in the ever-increasing service economy and to lower the entry barriers for new players (especially SMEs) who may not have the knowledge or resources to correctly negotiate and setup SLAs, monitor and enforce the delivery of performance as agreed before. As depicted in Figure 2, the Aleph Website represents an example for a next-generation, rich intermediary. It aims to increase Web Services transparency and to reduce the time potential service consumers have to spend to locate and procure services. Dedicated service crawlers automatically identify electronic services on the Web, while certain algorithms are used to filter the Web and index service specific features to enable comparison through unified presentation. Finally, the provision of tools for service bundling extends the intermediary's scope towards a central contact point for the improved user/services interaction.

Resource Consumption. In a future IoS, Web-based resources will be consumed by both the long tail of private users [8] and enterprises who consume business services. Especially in the professional 
context, the Internet of today is mostly only used to gather information, but not to seek and consume electronic services. Due to advancements in the underlying technology and the emergence of novel applications (which we will introduce below), companies will be able to interconnect their business functionality and automate information exchange with suppliers and customers in order to improve their productivity, the quality of their products and to allow for flexibility with regard to changing external requirements.

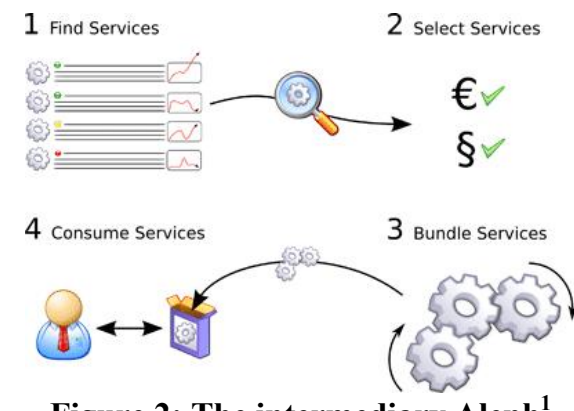

Figure 2: The intermediary Aleph ${ }^{1}$

\subsection{Applications}

Management. To realize the above mentioned reliability and predictability of electronic applicationto-application connections and to thus be able to effectively manage them, application interfaces must be attached formalized and standardized information about their functional as well as non-functional properties. In particular the formal parameterization of non-functional properties such as monetary cost for usage, compliance with certain standards, quality or security determinants helps to avoid unclear terminology and variability of electronic services and therefore enables the efficient establishment and enforcement of Service-Level Agreements (SLAs). In the course of the European Services Sciences, Management and Engineering-Initiative [11], new concepts for the exact, quantifiable formalization of non-functional service properties are currently being developed.

Resource Types. In the Internet of today, relatively static content or proprietary island applications represent the prevailing sort of resources offered to users. In the envisioned IoS, resources will become "richer". In this context, richness refers to several dimensions such as those proposed in [8]: First, a high degree of customizability will allow user to consume resources actively in the future Internet. The front-end of applications will become highly dynamic, enabling

\footnotetext{
${ }^{1} \mathrm{http}: / /$ aleph-webservices.com/
}

the customer to self-service in an intuitive fashion. Mash-up platforms as those presented in [2] represent first examples of interfaces that allow users to assemble, customize and operate modular applications according to their individual requirements. Applications will also become highly interactive. As argued in [1], contextual information (e.g., a user's personal preferences or the kind of mobile device she is currently using) ensure users are provided those Web-based resources which match best their proprietary needs. Third, up-to-date information and service functionality will represent a key benefit of resource provision in an IoS (currency). As discussed in the section focusing on technological aspects, novel protocols and design principles allow for the automated and fast dissemination of information in the Web. Last, instead of static content or simple functionality, more "heavy-weight", even enterprise class services that provide high information processing capabilities can be offered or consumed via the Web, enabled through the assurance of predictability and dependability.

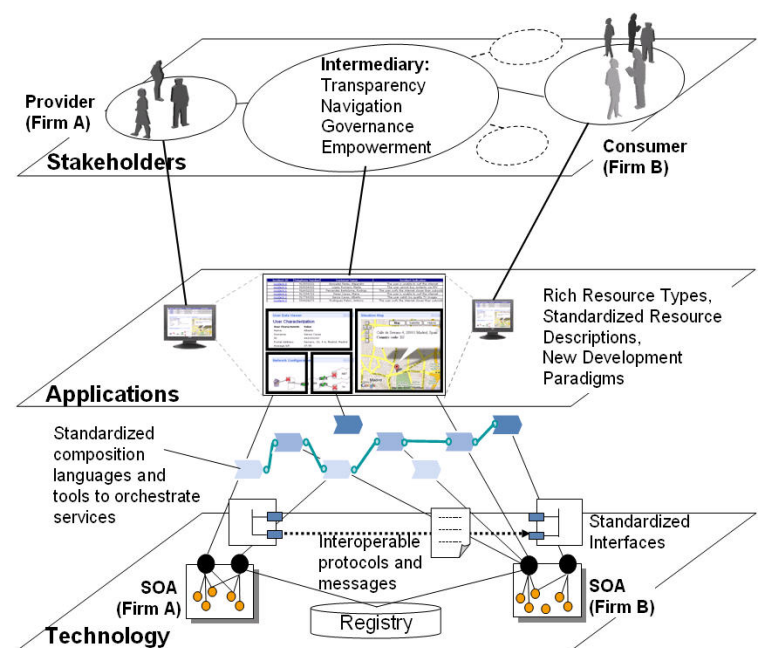

Figure 3: The Internet of Services facilitates user/service interaction and seamless crossorganizational collaboration

Development. In an IoS, novel approaches for the development of applications are expected to prevail. Rather than building static software artifacts that may be provided over the Internet, the envisioned IoS will rely on principles such as high end-user involvement, continuous change (O'Reilly promotes the end of the traditional software release cycle [3]) and the loose coupling of applications. A change of established practices, in particular with respect to Software Lifecycle (Traditional methodologies such as the V- 
model and the Rational Unified Process (RUP) will be replaced by more agile approaches such as Agile methods such as Xtreme Programming (XP)), Development Paradigms and Software Architecture (Lightweight programming languages and models support the fast and efficient setup and reuse of application components) and finally the Development Management Approach (end-users will be regarded as co-developers rather than passive consumers) will be required to cope with these challenges.

\subsection{Technology}

Service-oriented Architectures (implemented on the basis of the Web Services stack) as described above are about to emerge as prevailing technological foundation of the Internet of Service. The bottom layer of Figure 3 shows two different companies that expose certain services (black circles) which are possibly composed of other, internal services (red circles) and made publicly available via a central registry. Major extensions are now being deployed which represent essential enablers of a global SOA's take-up.

Composition. In the Internet of today, users may seek and retrieve information or functionality via navigators such as Google, but are usually not enabled to draw on more than one "resource" at a time. In the envisioned IoS, private users or businesses are expected to be able to select several out of a universe of interoperable Web-based resources and to compose them to realize required functionality. In this context, widely accepted and easily deployable orchestration languages such as BPEL are expected to prevail (The blue arrows in Figure 3 visualize the composition of different services across company boundaries in alignment with a given business process). Besides such languages which can only be handled by technical experts, different platforms and tools are currently about to emerge which also allow users with little technical expertise to compose resources from almost arbitrary locations in the Web: Google.com represents a first intuitive example for a so-called Mash-up platform as it allows users to aggregate and arrange different "gadgets" which represent either mere content (e.g., pictures, text, weather forecasts, Web cams) or certain application functionality (e.g., calculators). An intuitively usable gadget repository can be accessed and enables users to select and subsequently drag and drop some of the gadgets on their individual user interfaces. This kind of Mash-up-platform facilitates the mere aggregation of resources (which is also often referred to as syndication) but does not foresee any kind of interaction between them. Yahoo pipes ${ }^{2}$

\footnotetext{
${ }^{2}$ http://pipes.yahoo.com/
}

represent an example for a second category of Mashup platforms which are not limited to mere resource syndication: Information feeds (RSS-compliant) can be selected and visually aggregated; their respective content can be analyzed and filtered on the basis of arbitrary keywords; finally, the resulting pieces of information can be used to find related resources such as pictures or videos on websites such as Flickr ${ }^{3}$. Mash-up platforms like this are different from the ones assigned to the first category as they involve the processing of resources rather than their mere syndication. Still, resources are only loaded and processed at one point of time and do not interact in a stateful fashion similar to Web Services that are called according to a previously defined choreography. Only very few examples exist today that already try to automate the mashing of application functionality rather than their simple aggregation. The firm Kapow Technologies ${ }^{4}$ has recently published its vision of socalled Enterprise Mash-ups. It focuses on empowering users of their Web Integration Platform to integrate Web-based resources on several different levels and thus efficiently realize rapid portal creation, content migration, synchronization and syndication, composite application integration and SOA enablement. According to the firm's publications, logic-based Mash-ups can be setup on the basis of a merely visual, technology- and complexity hiding modeling interface. Enterprise applications which are encapsulated as, for example, Web Services or by REST-based interfaces can mashed up to build new functionality. In the near future, more advanced platforms are expected to emerge which allow for the stateful composition of rich services in the professional context. Remaining hurdles to achieve this include the wide agreement on standards for business process choreographies as well as structure and semantics of the messages exchanged in the course of these business processes [12].

Resource Interfaces. As argued above, the current Web comprises countless "information islands" and also applications that are mostly accessible via proprietary portals. Information is usually represented with the help of the widely accepted standard HTML that may feature some meta-information (e.g., for describing the respective Website's content) which is mostly not well standardized and therefore only hardly machine-readable, however. In an IoS, applications that are encapsulated as standardized services (possibly as Web Services which rely on WSDL as interface standard) are envisioned to become the prevailing form of application access. First developments in this

\footnotetext{
${ }^{3}$ http://flickr.com/

${ }^{4}$ http://www.kapowtech.com/
} 
direction can already be observed in the field of "information interfaces". Rather than publishing information in a static, html-based fashion, Website owners increasingly decide to encapsulate it as XMLbased files that comply with the Really Simple Syndication $\left(\mathrm{RSS}^{5}\right)$ standard which supports the fast aggregation of content from arbitrary sources in the Web. Besides the concept of standardized and easily usable encapsulation of information or application functionality, resources of all kinds are expected to be augmented by machine-readable semantics, making them accessible to intelligent queries and machinereasoning, allowing for a greater interoperability of information systems and reducing the mediation of human operators involved in information management. By formalizing the meaning of Web-based resources, the process of retrieving and dealing with the desired information or functionality can be significantly accelerated and improved. So far, resources are designed for consumption by human beings who may search (supported by navigators such as Google), request and read content or consume application functionality offered by a certain Website. Semantic annotations are based on representation metalanguages like $\mathrm{RDF}^{6}$, RDF Schema and OWL. RDF Schema is a vocabulary for describing properties and classes of RDF-based resources, including semantics for the generalization of hierarchies of such properties and classes. Ontologies extend this concept by providing conceptualizations created by domain experts, and are usually described using $\mathrm{OWL}^{7}$ (which adds relevant vocabulary for describing properties and classes: among others, relations between classes (e.g. disjointness), cardinality (e.g. "exactly one"), equality etc.). In this context, Semantic Web Services (SWS) enrich traditional Web Service technology by semantic annotations, providing the framework for automated discovery and composition of program functionality. European research has yielded the leading conceptual SWS framework $\mathrm{WSMO}^{8}$, a family of respective representation languages $\left(\mathrm{WSML}^{9}\right)$, and the prototype implementation of a WSMO-compliant execution environment WSMX.

All efforts in the Semantic Web community have so far focused on formalizing Web-based resources to simplify their retrieval, reasoning and handling based on machines. The wide acceptance of certain standard ontologies and the integration of human users represent the two major challenges for the future in

\footnotetext{
${ }^{5}$ http://www.rssboard.org/rss-specification

${ }^{6} \mathrm{http}: / / \mathrm{www} . \mathrm{w} 3.0 \mathrm{rg} / \mathrm{RDF} /$

${ }^{7}$ http://www.w3.org/TR/owl-features/

${ }^{8} \mathrm{http} / /$ www.w3.org/Submission/WSMO/

${ }^{9} \mathrm{http}: / /$ www.wsmo.org/wsml/
}

this field. "Semantic islands" are only useful to a limited degree. The inclusion of human users can be achieved by interconnecting user-driven, lightweight ontologies (often referred to as folksonomies) with more formal ones (e.g., OWL) on the one hand and by making machine-based reasoning easily and intuitively accessible to human users.

Messaging protocols. In today's Internet, information islands and proprietary application functionality represent the prevailing sort of resources. Applications are only rarely interconnected to exchange messages according to defined protocols, partly as a consequence of a lack of easily usable standards. Some companies have encapsulated their services with the help of electronic interfaces which are often extremely heterogeneous, however [13]. In the enterprise context, point-to-point message exchange interfaces may thus exist (e.g., on the basis of the UN/EDIFACT standard), but they are of limited reach [8] since neither message sequences, nor message structure or semantics underlie uniform standards.

In an IoS, resources can be composed to exchange data on the basis of easily usable and widely accepted standards. The Simple Object Access Protocol (SOAP) specifies a data format of the messages to be sent between service providers and requesters and provides a basic messaging framework that more abstract layers can build upon. The Representational State Transfer (REST) is an architectural style that has been proposed by Fielding in his doctoral dissertation [14]. With the help of REST, Web clients can interact with arbitrary Web resources (sources of specific information) in a uniform way and thereby exchange XML-based messages. Resources are uniquely addressable by means of a global identifier (URI) and support a well defined and also constrained set of supported operations (so-called HTTP verbs such as GET, PUT). By using resource identifiers and HTTP verbs together, clients can invoke REST-based services and request the delivery of, for example, an XML-file containing the desired specific information.

\section{Global industrialization of information intensive services through the IoS}

In most developed countries' economies, the services industries, especially businesses dealing with information intensive services, have an increasingly relevant share of the GNP. However, services performance is low compared to traditional manufacturing industries. They suffer from high variability, informal terminology and unquantifiability; recognized and comprehensive frameworks for information service performance measurement are 
rarely applied and effective technological support for information service industries is mostly limited to local applications but does not yet span across company boundaries. Therefore, new concepts are required to improve productivity and foster the industrialization of information intensive services.

\subsection{Industrialization of traditional product manufacturing}

The term industrialization is used in various contexts and with very different meanings. In this work we refer to industrialization as the process of applying scientific means to be able to formalize, standardize and automate the creation of customer value with the final goal to improve performance (measured on the basis of the indicators productivity, quality and flexibility) [15]. The industrialization of traditional product manufacturing (Figure 4) can trace its roots to the work of inventors such as James Watt in the $18^{\text {th }}$ century who developed techniques to make steam engines significantly faster, safer, and more fuelefficient than existing devices. During the $18^{\text {th }}$ and also the $19^{\text {th }}$ century, the general focus of industrialization was mainly on the development of power machinery, interchangeable parts, and the division of labor. A more holistic perspective that takes into account overall production processes and also the management of the single works did not emerge until the early $20^{\text {th }}$ century:

\begin{tabular}{|c|c|c|}
\hline $\begin{array}{c}\text { 19th century } \\
\text { Whitney, Bessemer }\end{array}$ & $\begin{array}{l}\text { Early 20th century } \\
\text { Taylor, Ford, Sloan }\end{array}$ & $\begin{array}{l}\text { Late 20th century } \\
\text { Ohno, Krafcik }\end{array}$ \\
\hline $\begin{array}{l}\text { - Power machinery, } \\
\text { interchangeable parts, } \\
\text { division of labor } \\
\text { - However, no } \\
\text { interrelation of different } \\
\text { processes, no } \\
\text { consideration of the } \\
\text { single workers }\end{array}$ & $\begin{array}{l}\text { - Holistic view on } \\
\text { production processes } \\
\text { - Introduction of the } \\
\text { „production-line } \\
\text { approach“ } \\
\text { - Separation of process } \\
\text { planning and execution } \\
\text { - Worker specialization } \\
\text { - Focus on both } \\
\text { productivity and flexibility } \\
\text { (Sloan) }\end{array}$ & $\begin{array}{l}\text { - Lean Manufacturing to } \\
\text { reduce waste } \\
\text { (superfluous overhead) } \\
\text { - Simultaneous } \\
\text { improvement of } \\
\text { "productivity } \\
\text { "quality } \\
\text { "flexibility }\end{array}$ \\
\hline
\end{tabular}

Figure 4: The industrialization of traditional product manufacturing

Taylor [16], Henry Ford and Alfred P. Sloan introduced scientific means to the analysis of production processes, promoted the production-line approach and focused on both productivity and manufacturing flexibility. In the second half of the $20^{\text {th }}$ century, the first "Lean" manufacturing systems emerged [15]. As opposed to the traditional, buffered approach (where inventory levels are high, assembly lines have built-in buffers against partial system breakdowns and utility workers are kept on the payroll to buffer against unexpected demand), the Lean
Manufacturing approach aims to reduce superfluous overhead (represented by the seven kinds of "waste": defects, overproduction, excessive inventory, transportation, waiting, motion, over-processing). In this way, all relevant performance indicators (productivity, quality, flexibility) shall be improved.

\subsection{Emerging industrialization of information intensive services}

Today, service businesses suffer from high variability, unclear terminology and unquantifiability of performance parameters or input/ output values. The above presented IoS reference architecture bears great potential to support the "Lean" implementation and management of information intensive services and to thus improve productivity, flexibility and quality of these services businesses. The following paragraph will provide an overview of how the IoS facilitates the reduction of the seven major kinds of waste targeted by the Lean Manufacturing approach:

Waste of defects: Service consumers desire reliable and accurate information. Today, proprietary interfaces and application interconnections bear the risk of processing errors. Semantics of messages as well as business process choreographies need to underlie widely accepted standards to avoid manual mapping effort. The IoS will stride along with a high degree of standardization with respect to service interfaces, message structure and semantics. In this way, manual mapping of data or the setup of proprietary interface connectors is made superfluous, thereby increasing information service quality and productivity.

Overproduction and Excessive Inventory: Similar to the manufacturing industry, service businesses deal with inventory (all the service process steps that have been performed and stored before the customer arrives) which enables to buffer value creation from the variability of demand and to benefit from economies of scale. On the other hand, inventory must be reduced to a minimum as it introduces costs for storage and maintenance. To retain a minimum level of inventory and to avoid overproduction, the "push-pull boundary" [17] must be easily adjustable. The IoS allows for an on-demand environment as electronic services can easily be retrieved, customized, assembled and consumed without requiring prior mapping or huge programming efforts. Overproduction can be limited through high transparency, the application of formal rules and the exact implementation of an ondemand environment. In this way, the execution of process steps ahead of demand can be avoided.

Transportation: In the manufacturing context, the transportation of goods is regarded as an activity that 
must be reduced to a minimum as its does not add customer value but cost time and bears the risk that goods are lost or damaged. In the field of information services, the transportation of bits in different formats from one proprietary application or database to another can be considered an equally worthless activity. The envisioned IoS will reduce the time required for transport to a minimum since electronic services are capable of quickly and seamlessly exchanging uniformly structured information. Also, information transportation routes will follow clear, previously defined and optimized ways: The orchestration of services according to a dedicated business process will help avoiding time-consuming routing decisions.

Waiting: The idle time between single processes, leading to human resources or equipment that do not operate at full capacity represent another kind of waste. The next-generation Internet will provide managers the possibility to measure service load, create and evaluate statistics and adjust service choreographies such that idle times for each of them are minimized.

Waste of motion: In manufacturing, ineffective work procedures represent one of the causes for manual efforts that do not add value from a customer's point of view. The dawning IoS has the potential to reduce unnecessary process steps as it provides workers a technical means which structures, simplifies and optimizes the processing of information.

Over-processing: Over-processing, induce for example by over-fulfillment of customer requirements, may result in useless consumption of resources such as time or inventory. As described above, the envisioned IoS relies on independent institutions which account for precise negotiation and enforcement of SLAs that define the performance metrics to be delivered by a service provider. In this way, over-processing in the information service industry can be strongly reduced.

\section{Conclusion}

Today, information intensive services still suffer from high variability, hard quantifiability and informal terminology. However, these properties do not represent inherent characteristics, but major hurdles which need to be taken down on the path to a global industrialization. We propose a novel, holistic reference architecture that is capable to drive information service industrialization: The envisioned Internet of Services revolutionizes the Internet as of today with regard to the three dimensions stakeholders, applications and infrastructure and shows great potential to support the "Lean" management of information intensive services. On the basis of technical case studies, we substantiated the hypothesis of this emerging new generation of the Internet and also elaborated on remaining hurdles for its successful adoption.

\section{References}

[1] C. Schroth, T. Janner: Web 2.0 and SOA: Converging Concepts Enabling the Internet of Services, IT Professional, Vol. 9, No. 3, IEEE Computer Society, 2007

[2] C. Schroth, O. Christ: Brave New Web: Emerging Design Principles and Technologies as Enablers of a Global SOA, in Proceedings of the 2007 IEEE International Conference on Services Computing (SCC 2007), Salt Lake City, USA, 2007 [3] T. O'Reilly: What is Web 2.0, available online at: http://www.oreillynet.com/pub/a/oreilly/tim/news/2005/09/3 0/what-is-web-20.html

[4] R. Högg, M. Meckel, K. Stanoevska-Slabeva, R. Martignoni: Overview of business models for Web 2.0 communities, Proceedings of GeNeMe 2006, p. 23-37, Dresden, 2006

[5] G. Alonso, F. Casati, H. Kuno, V. Machiraju: Web Services Concepts, Architectures and Applications, in: Springer, 2004

[6] M. MacKenzie et al: OASIS - Reference Model for Service Oriented Architecture 1.0, http://www.oasisopen.org/committees/tc_home.php?wg_abbrev=soa-rm

[7] P. Wainewright. Semantic integration, http://www.looselycoupled.com, 2003

[8] P. Evans, T. S. Wurster: Blown to Bits: How The New Economics of Information Transforms Strategy, Harvard Business School Press, Boston, MA, USA, 2000

[9] D. C. North: Institutions, Institutional change and economic performance, Cambridge University Press, Cambridge, 1990

[10] P. Weill, J.W. Ross: IT Governance: How Top Performers Manage IT Decision Rights for Superior Results, Harvard Business School Press, Boston, MA, USA, 2004

[11] IBM: Service Sciences, Management and Engineering. available online at: http://www.research.ibm.com/ssme/index.shtml, 2007 [12] C. Schroth, T. Janner, G. Stuhec: UN/CEFACT ServiceOriented Architecture: Enabling Both Semantic And Application Interoperability, Proceedings of the Symposium "Communication in Distributed Systems" (KiVS), Workshop: Service-Oriented Architectures und ServiceOriented Computing, Bern, Switzerland, 2007

[13] European Commission: The European e-Business Market W@tch. available online at: http://www.ebusinesswatch.org/, 2007

[14] R.T. Fielding: Architectural styles and the design of network-based software architectures, Ph.D. thesis, University of California, Irvine, 2000

[15] J. F. Krafcik: Triumph of the lean production system, Sloan Management Review, Vol. 30, No. 1, pp.41-52, 1988

[16] F. W. Taylor: The Principles of Scientific Management, Harper Bros, New York, 1911

[17] S. Chopra, M.A. Lariviere: Managing Service Inventory to Improve Performance, MIT Sloan Management Review, Vol. 47, No. 1., 2005 